\title{
Reliability analysis and assessment of the explosion risk in a hybrid collector
}

\author{
S. Copelli ${ }^{1}$, E. C. Rada ${ }^{2}$, M. Ragazzi ${ }^{2}$, V. Torretta ${ }^{3} \&$ M. Schiavon ${ }^{2}$ \\ ${ }^{1}$ Department of Science and High Technology, \\ Insubria University of Varese, Italy \\ ${ }^{2}$ Department of Civil, Environmental and Mechanical Engineering, \\ University of Trento, Italy \\ ${ }^{3}$ Department of Biotechnologies and Life Sciences, \\ Insubria University of Varese, Italy
}

\begin{abstract}
Dust removal from a gas stream is a common problem in many industrial processes (e.g. power, cement, steel plants, etc.). In the last decades, plant managers more and more often have had to face the necessity of improving de-dusting systems in order to meet more stringent law requirements, inspired by the principle of the "maximum safety technologically feasible", but also pushed by the increased penetration of environmental issues into public opinion, and its meaning in terms of company image. Budgets for these improvements play a role in a general asset management strategy: de-dusting, being often an operation having low influence on production performances, is seen more as a "necessary cost" than a profit generator. The trend for companies, especially in the actual economical scenario, is to reuse existing de-dusting plants, enhancing their efficiencies rather than install new plants that would involve costs for the decommissioning of obsolete equipment. The obvious economic benefits of retrofits are counterbalanced by technical disadvantages. In particular, a systematic assessment of safety issues is required, not limited to the safetyoriented design of new machines, but extended in the evaluation of the impact that changes in process conditions (induced by the new equipment) can have on the existing ones.

In this work, explosion problems in the de-dusting section of a cement plant have been considered. Particularly, using fault tree analysis, it has been evaluated
\end{abstract}


the changing in the overall risk (considering one year of mission time) of explosion referring to a hybrid-like collector realised by introducing a Fabric Filter (FF) downstream with respect to an Electrostatic Precipitator (ESP). Results have shown that a chain of failures in the FF section may affect relevantly the risk of explosions occurrence in the collector leading to the unavoidable need for the introduction of mitigation actions into the system.

Keywords: cement plants, risk assessment, explosions, safety, hybrid collectors, retrofit.

\section{Introduction}

Cement manufacturing is one of the largest mineral commodity industries in the United States, with an estimated production capacity of greater than 73 million tons annually [1]. On the contrary, in European countries, a huge decrease in cement consumption has been registered during the last five years. Particularly, in 2012, there has been a $22.1 \%$ (with respect to 2011) decrease in the manufacturing of cement and this trend seems to continue [2].

The new regulation on Solid Recovery Fuel (SRF) from municipal solid waste (MSW) can change this trend and give a higher speed to this sector and decreasing the dust problem but increasing the chlorine or the heavy metals ones [3-6].

Essentially, the most important part of a cement manufacturing process is the kiln, a horizontal steel cylinder, lined with firebrick and slightly sloped downward from the raw material feed to the fuel feed supply. Typically, a kiln rotate slowly (maximum 1-3 rpm), heating the raw material mix to obtain very hot, marble-sized chunks known as "clinker". Once out of the kiln, the clinker undergoes a cooling process. When sufficiently cool, the clinker is ready to pass through a series of grinding and milling processes providing that gray powder known as cement.

The cement industry is very capital intensive. Some of the major pieces of equipment include pulverisers, dust collectors, electrostatic precipitators, and rotary kilns. Energy costs can account for up to $40 \%$ of the total cost of cement manufacturing. Currently, over $90 \%$ of the installed capacity uses coal as primary fuel for the kiln burner. Conversion to coal was started in the 1970s. Unfortunately, the use of coal as fuel is very dangerous because of the possible triggering of fire and explosions during cement manufacturing.

Moreover, the cement industry has to face another relevant problem: atmospheric dust emissions. These emissions became in the last years the most studied problem in terms of urban pollution independently if they are produced from industrial processes, from traffic or from cooking or domestic heating [7$10]$.

Dust removal from a gas stream is a common and quite cheap operation required in many industrial processes but, in the last decades, more and more stringent law requirements have forced this common operation to become very expensive. Budgets for introducing improvements (or new units) in the already existing de-dusting equipment play a fundamental role in the general asset 
management strategy: in fact, de-dusting, being often an operation having low influence on production performances, is unavoidably seen more as a "necessary cost" than a profit generator. Companies' trend, especially in the actual economical scenario, is to reuse existing de-dusting plants, enhancing their efficiencies rather than install new plants that would involve costs for the decommissioning of obsolete equipment. Unfortunately, the obvious economic benefits of retrofits are counterbalanced by technical disadvantages and possible safety related problems. In particular, a systematic assessment of safety issues, not limited to the safety-oriented design of new machines, but extended in the evaluation of the impact that changes in process conditions (induced by the new equipment) can have on the existing ones, must be done.

In this work, we have focused on the relevant problem of the triggering of explosions in the de-dusting section of a cement plant. Particularly, using fault tree analysis, it has been evaluated the changing in the overall risk (considering one year of mission time) of explosion referring to a case study where a hybridlike collector is realised by introducing a Fabric Filter (FF) downstream with respect to an already installed ElectroStatic Precipitator (ESP). Results have shown that failures in the FF section may affect relevantly the risk of an explosion occurrence in the collector itself leading to the unavoidable need for the introduction of mitigation actions into the system.

\section{Description of the hybrid-like collector}

Hybrid collectors are abatement systems that have been designed specifically to take into account all the factors affecting their operational efficiency. Because of this fact, they have very high collection efficiency, and they represent the best technology currently available in order to fully comply with the limit values laid down by specific authorizations issued by the competent bodies.

The hybrid collection system treated in this case study has been realised by a retrofit operation consisting of an addition of a fabric filter downstream an already installed electrostatic precipitation section.

Given the particular system design, the amount of dust emitted into the atmosphere is considerably lower than that allowed by the strict environmental standards in force.

Hot gases (about $300^{\circ} \mathrm{C}$ ), coming from the pre-heating section of the kiln, are partly used to dry and ventilate both the coal and the crude feed mills but, while the gases of the coal mill are filtered independently, the dusty gases used in the kiln are mixed with the gas coming from the pre-heater in a tower (called "conditioner") where they are cooled (till about $160^{\circ} \mathrm{C}$ ) and humidified with water spray; the amount of dosed water is regulated by a computer as a function of the gas temperature.

Then, the conditioned gases enter the electrostatic section of the hybrid filter, whose design is such as to allow a very low gas velocity (in order to favour the separation of the dusts from the gaseous stream). The operating principle of an electrostatic precipitator is based on the effect of ionization of a neutral gas (corona effect) when it passes through a strong electric field formed by issue 
(negative polarity) and precipitation (or collector) electrodes (positive polarity). Thanks to the very high applied voltage (in this case, $65 \mathrm{kV}$ ), the issue electrodes emit electrons by photoelectric effect and charge the dust particles of which the gas to be de-dusted is rich; in this way, the charged dust particles will be attracted by the precipitation electrodes (or plates). The dust settles on the plates so that, periodically, they have to be "shocked" in order to be kept clean and fully operative. Particularly, an electrostatic precipitator can remove dust particles as small as $1 \mu \mathrm{m}$ with an efficiency exceeding $99 \%$.

Immediately then, the partially de-dusted gases pass through another section: the fabric filter.

A fan located downstream of the fabric filter aspires the air flow to be dedusted, causing its entry in the lower part of the filter; hence, the gas going up meets the rows of bags and passes from the outside to the inside of their tissue cylinder.

The particles suspended in the gas are retained on the outside of the bags thanks to both the structure of the fabric fibers and a layer of particulate laying outside the fabric of each bag (which is capable of retaining even the finer particles).

The collapse of each bag on itself, given by the outside-inside air flow, is prevented by a basket that keeps roughly the shape of a tube.

The de-dusted air inside of each bag is now aspired and removed by the filter in the upper part of the chamber to be sent to the chimney, always thanks to the fan cited above. Approximately every $8 \mathrm{~min}$, in order to avoid the clogging of the filtering surface because of an excessive deposit of dusts, an air jet at high speed is blown inside of each bag: such a jet, impacting against the filtering surface, causes a sudden expansion of the bag and allows for the detaching of the crust of dust that has been formed on it. This cleaning system is called "pulsed jet" and has the advantage of not having to exclude the bags from the filtration system during cleaning operations; the air jet is provided instantly through solenoid valves from dedicated storage tanks, whose operating pressure is about 6 bar, and acquires a high speed through the venturi ducts placed on the basket of each bag. To maintain continuous filtration during the cleaning operation, the air is fed to a row of bags at a time, allowing the other rows to continue to carry out their activity. The detached material falls into the hoppers at the bottom of the filtering chamber and, then, it is extracted by means of a worm screw and returned to the kiln by means of conveyor belts.

All these constitutive parts of the plant have been reported in fig. 1, which shows a simple sketch of the de-dusting unit.

\section{Risk assessment in cement plants}

\subsection{General risk assessment procedure}

When talking about Quantitative Risk Analysis (QRA), we referred to as a set of methodologies and structured procedures having the aim of determining (both qualification and quantification) the so-called "risk function", which is a fundamental tool in a decision making process. 
The estimate of the risk is generally performed starting from statistical data derived from the history of accidents occurred in plants similar to the one under study: in this case, accidents in cement plants. On the contrary, the determination of the consequences of accidental events involving the plant under study depends very closely on the location of the industrial site (at least for what concerns the aspects of meteorology and hydrology, population distribution, etc.) and, therefore, it is generally necessary an adaptation of the statistical data to the particular plant.

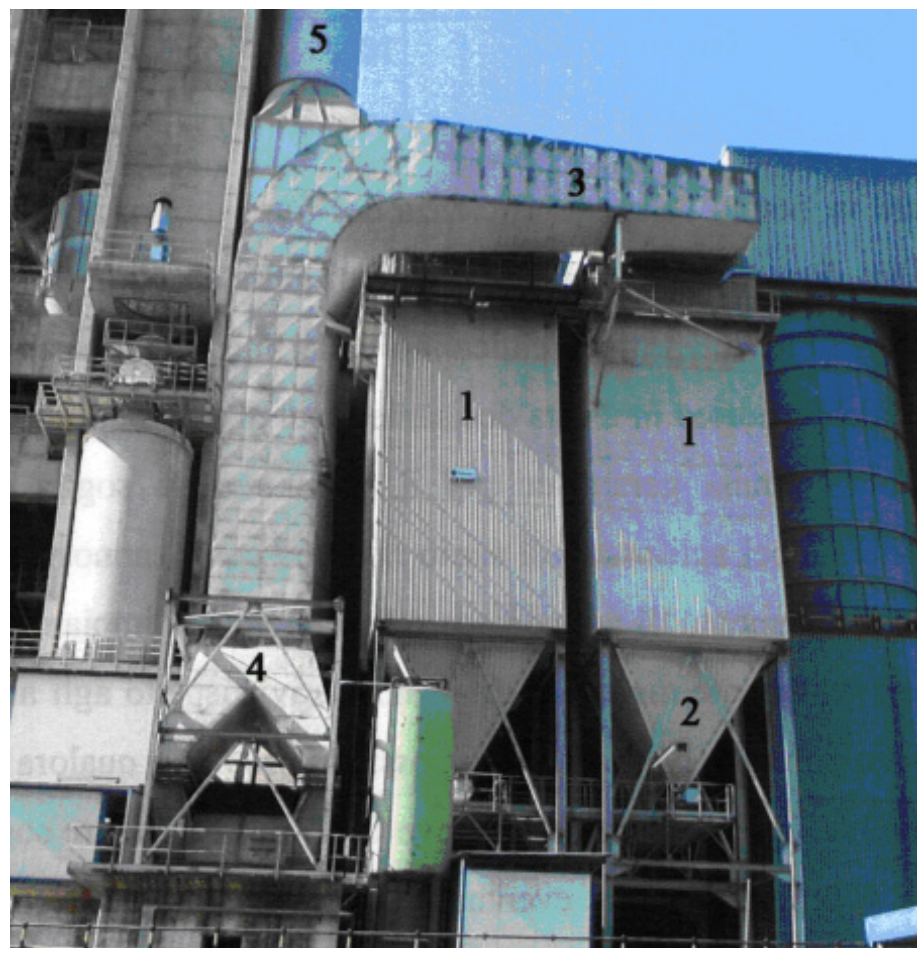

Figure 1: Sketch of the case study hybrid collector. 1) Final sections of the fabric filters; 2) hoppers; 3) de-dusted air duct; 4) fan; 5) bottom of the chimney. The ESPs are located behind the fabric filters.

In most cases, the risk linked with whatever industrial activity results from the use of chemicals or other potentially hazardous materials [11,12]. Therefore, the unavoidable starting point of a risk analysis is always the so-called "hazards identification" procedure. Protection systems installed into the plant in order to prevent or, at least, limit the possible effects of accidental events, must to be taken into account during this evaluation step. In fact, it is important to specify that an accidental event can occur only if, at the same time, there is a process anomaly (caused by a variety of reasons) and the failure of all the related protection systems occurs. 
The next step of a risk analysis is the determination of all accidental scenarios associated with the previously identified hazards, possibly filtered by the effect of the available protection systems.

Then, considering all the phenomena involved in an accident (both physical and chemical), it is possible to evaluate its effects on environment, workers, people living in the neighbouring of the facility, etc., employing the procedure constituted by the following steps:

a) identification of all the events that may contribute to the occurrence of that accident;

b) estimation of the probability of occurrence of such events and their consequences;

c) quantitative determination of the risk function related to the considered accidental event (and its use for decision making).

It is important to underline that, in order to use such a procedure, a thorough knowledge of the considered industrial plant and its processes has to be acquired for the search of those failures that may trigger an accident (initiating events).

For what concerns the estimation of the probability of occurrence of an accidental event, it is possible to employ dedicated techniques such as fault tree analysis.

Finally, after determining the probability of occurrence of each identified accidental event, the integration of all results allows for the determination of the function associated with the risk linked to the activity in question.

\subsection{Fault tree analysis for QRA}

Fault tree analysis is a widely used technique capable of both determining all the credible modes of occurrence of an undesired event (called, top event), caused by a complex concatenation of other events (qualitative analysis), and estimating the frequency of occurrence of the undesired event on the basis of the frequency of occurrence of the events that cause it (quantitative analysis [13]).

It is a structured procedure that requires a deep knowledge of all the components of the analysed system; particularly, the chain of intermediate events leading to the occurrence of the top event must be decomposed (through the use of logic gates) until reaching the "roots" of the tree, which are constituted by the so-called "primary events", that are events for which the probability of occurrence are always known (e.g., from databases).

Therefore, going from the bottom (roots or primary events) to the top (top event) of a fault tree, it is possible to quantify the so-called reliability function, $R(t)$, of a system, that is the probability that, in correspondence of an allotted time interval (also called mission time, $t$ ), a system performs properly the function for which it was built. From the $R(t)$ function, it is also possible to calculate both the unavailability function, $q(t)$, which is the probability that the system is not able to perform its function (because it is broken) at time $t$, and the frequency of occurrence, $W(t)$, which is the number of times that the system is not expected to be able to perform its function in the mission time. Of course, before quantifying a fault tree, it is necessary to determine these quantities for all 
primary events that have been identified in the construction of the fault tree itself.

Then, it is possible to quantify the entire tree by using a variety of methods. One of the most simple and known is the Minimal Cut Sets (MCS) method. Particularly, a MCS is the minimum combination of primary events which is necessary and sufficient to ensure the occurrence of the top event; in other words, the top event occurs if all the events in a MCS occur simultaneously. Therefore, the unavailability of a MCS coincides with the probability of occurrence of the top event due to the considered MCS. Since the individual events involved in a MCS are all independent, the overall probability that all events occur simultaneously is given by the following relation:

$$
q(t)=\prod_{i=1}^{n} q_{i}(t)
$$

where $n$ is the order of the MCS (the number of events that involves the MCS) and $q_{i}(t)$ is the unavailability of each component of the MCS.

A top event can occur if anyone of the MCS that can cause it occurs. The likelihood of the top event in a given interval of time (mission time, $T$ ) can thus be calculated as the union (logical OR) of the probability of occurrence of each MCS that can cause the top event itself, using the following relation.

$$
W(t)=\int_{o}^{T}\left[\sum_{i=1}^{n}\left(\left(\prod_{\substack{j=1 \\ j \neq i}}^{n} q_{j}(t)\right) \lambda_{i}\right)\right] d t
$$

where $\lambda$ is the failure rate, that is, the frequency with which a system fails (or the fraction of the components that fail per unit of time, $\mathrm{y}^{-1}$ ) and $t$ is time, $\mathrm{y}$.

As it is well known, the quantification of a fault tree is always an operation valid for orders of magnitude. It follows that no unnecessarily high accuracy is required in the estimate of the probabilities of occurrence of the primary events.

\section{Case study: de-dusting system retrofit}

In accordance with the purposes of the present research, the authors focused on the relevant problem of the triggering of explosions in the de-dusting section of a cement plant. Using fault tree analysis, it has been evaluated the changing in the overall risk (considering one year of mission time) of explosion referring to the case study where a hybrid-like collector is realised by introducing a Fabric Filter (FF) downstream with respect to an already installed ElectroStatic Precipitator (ESP).

The drafting of the fault trees concerning the top event "occurrence of an explosion in the de-dusting section" both before (ESP) and after (HYBRID FILTER $=\mathrm{ESP}+\mathrm{FF})$ the retrofit operation was taken into account. 
For what concerns the layout of the de-dusting section (before and after) and its functioning, reference has been made to the plant sketch reported in figure 1 .

Figure 2 shows the graphical representation of a fault tree realised for the plant configuration before the retrofit operation, that is, considering the dedusting section of the plant as constituted by the ESP (and its related instrumentation) only. Observing the tree, it can be derived clearly how an explosion of the ESP can be triggered as a consequence of different chains of events (all intended as failures).

In particular, the top event takes place in the case where both of the following final intermediate events occurs:

- E10: development of an overpressure higher than 6 bar inside the ESP chamber;

- E20: failure of the rupture disk installed on the ESP.

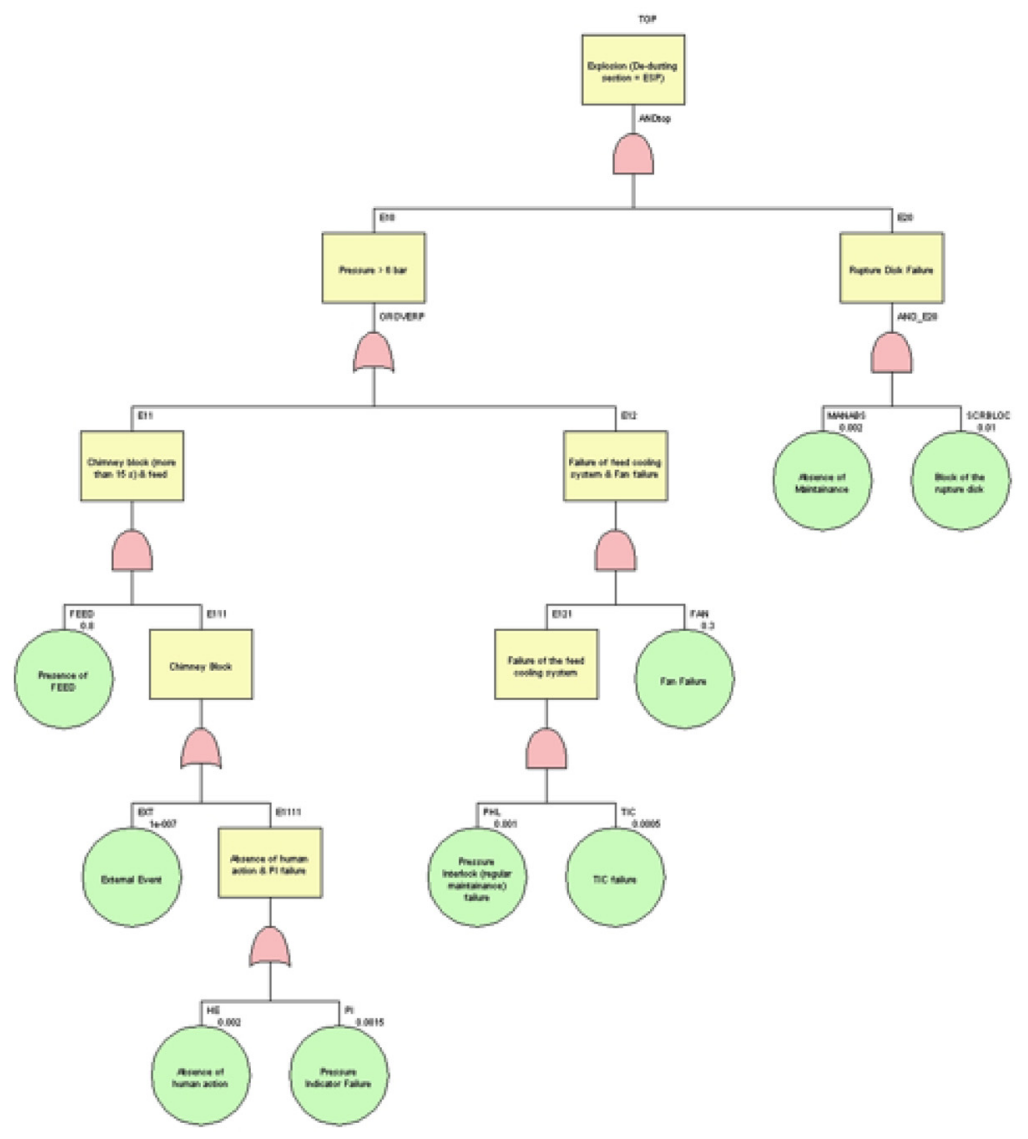

Figure 2: Fault tree for the top event "explosion in the de-dusting section" evaluated before the plant retrofit. 
It is important to state that, within the scheme of the fault tree, all the protective devices present into the plant section object of the analysis have been considered.

Performing the MCS analysis, it is possible to observe that there are $4 \mathrm{MCS}$ : 3 of order 4 and 1 of order 5 . Among the MCS of order 4, we have all the combinations of events in which, due to the presence of external factors causing the blockage of the chimney (EXT), the feed to the de-dusting unit (FEED), the absence of a scheduled and correct maintenance activity (MANABS), the blockage of the rupture disk installed on the ESP (SCRBLOC), the absence of a human action as a consequence of the sounding of the high pressure alarm (HE) and the breakdown of the ESP pressure indicator (PI), a pressure overcoming the maximum threshold value can be reached causing a physical explosion of the ESP chamber.

For what concerns the MCS of order 5, it involves the following combination of events: fan breakdown (FAN), absence of a scheduled and correct maintenance activity (MANABS), block of the rupture disk installed on the ESP (SCRBLOC), pressure interlock (PHL) and temperature controller failure (TIC) on the water spray feeding line that constitutes the cooling system available for the hot dusty gases exiting the kiln and addressed to the de-dusting system.

In order to determine all failure rates and unavailabilities, Dossier Ambiente manuals [4] have been used. Table 1 contains all the data necessary for the quantification of the fault tree.

Table 1: Database for failure rates, repair rates and unavailabilities. Case study: before the plant retrofit.

\begin{tabular}{|c|c|c|c|}
\hline $\begin{array}{c}\text { Primary event } \\
\text { code }\end{array}$ & $\begin{array}{c}\text { Failure rate } \\
{\left[\mathbf{y}^{-1}\right]}\end{array}$ & $\begin{array}{c}\text { Repair rate } \\
{\left[\mathbf{y}^{-1}\right]}\end{array}$ & $\begin{array}{c}\text { Unavailability } \\
{[-]}\end{array}$ \\
\hline EXT & - & - & $1 \cdot 10^{-7}$ \\
\hline FEED & - & - & $8 \cdot 10^{-1}$ \\
\hline MANABS & - & - & $2 \cdot 10^{-3}$ \\
\hline SCRBLOC & - & - & $1 \cdot 10^{-2}$ \\
\hline HE & - & - & $2 \cdot 10^{-3}$ \\
\hline PI & $3 \cdot 10^{-1}$ & 200 & $1.5 \cdot 10^{-3}$ \\
\hline FAN & - & - & $1 \cdot 10^{-2}$ \\
\hline PHL & - & - & $1 \cdot 10^{-3}$ \\
\hline TIC & $1 \cdot 10^{-1}$ & 200 & $5 \cdot 10^{-4}$ \\
\hline
\end{tabular}

Quantifying the fault tree using a simple numerical probability calculation involving the computation of all MCS, a value of the probability of occurrence of the top event equal to $5.610^{-8} \mathrm{y}^{-1}$ has been found. Such a value can be located in the acceptability zone for what concern both individual and societal $(<100$ inhabitants) risk. Therefore, just from this analysis, the system does not require to be integrated with redundant protection devices in order to improve the reliability of the de-dusting section.

Successively, the authors proceeded with the generation of the fault tree concerning the top event "occurrence of an explosion in the de-dusting section", 
now constituted by a fabric filter installed after the exit of the already existing ESP (that is, considering a retrofit operation).

In this case, the generated fault tree is too wide to be reported here but it is possible to briefly summarise the main results arising from its detailed analysis. Particularly, 7 different minimal cut sets can be observed: 3 of order 3, 3 of order 4 and 1 of order 5 .

Among the MCS of order 3, quite dangerous, we have all the combinations of undesired events and failures in which, due to the absence of a regular and correct maintenance activity on the rupture disks of both the fabric filter and the electrostatic precipitator (MANABS), the blockage of the rupture disks (SCRBLOC), the breakdown of the cleaning air timing system (TEMP), the absence of cleaning air (NOAIR) and the failure in closed position of the exiting valve of the fabric filter (VALCLO), a pressure overcoming the maximum threshold value can be reached causing a physical explosion of the hybrid -like collector.

Among the MCS of order 4, we have all the combinations of events previously determined for the ESP unit alone (even if with some little variants): blockage of the chimney (EXT), presence of feed to the de-dusting unit (FEED), absence of maintenance activity (MANABS), block of the installed rupture disks (SCRBLOC), absence of human actions even if a high pressure alarm is sounding (HE) and breakdown of the hybrid filter differential pressure indicator (PI). Finally, regarding the MCS of order 5, we can observe that it is the same involved in the plant configuration before the retrofit operation (this is because it involves only failures of equipment located upstream of the ESP and the fan).

As previously done, to determine all failure rates and unavailabilities, Dossier Ambiente manuals [14] have been used. Table 2 contains all the data necessary for the quantification of the new fault tree.

Table 2: Database for failure rates, repair rates and unavailabilities. Case study: after the plant retrofit.

\begin{tabular}{|c|c|c|c|}
\hline $\begin{array}{c}\text { Primary } \\
\text { Event Code }\end{array}$ & $\begin{array}{c}\text { Failure Rate } \\
{\left[\mathbf{y}^{-1}\right]}\end{array}$ & $\begin{array}{c}\text { Repair Rate } \\
{\left[\mathbf{y}^{-\mathbf{1}}\right]}\end{array}$ & $\begin{array}{c}\text { Unavailability } \\
{[-]}\end{array}$ \\
\hline EXT & - & - & $1 \cdot 10^{-7}$ \\
\hline FEED & - & - & $8 \cdot 10^{-1}$ \\
\hline MANABS & - & - & $2 \cdot 10^{-3}$ \\
\hline SCRBLOC & - & - & $1 \cdot 10^{-2}$ \\
\hline HE & - & - & $2 \cdot 10^{-3}$ \\
\hline PI & $3 \cdot 10^{-1}$ & 200 & $1.5 \cdot 10^{-3}$ \\
\hline FAN & - & - & $1 \cdot 10^{-2}$ \\
\hline PHL & - & - & $1 \cdot 10^{-3}$ \\
\hline TIC & $1 \cdot 10^{-1}$ & 200 & $5 \cdot 10^{-4}$ \\
\hline NOAIR & - & - & 0.1 \\
\hline TEMP & - & - & 0.05 \\
\hline VALCLO & - & - & 0.05 \\
\hline
\end{tabular}


Quantifying the fault tree using a classical numerical probability calculation involving the computation of all MCS, a value of the probability of occurrence of the top event equal to $3.8^{*} 10^{-6} \mathrm{y}^{-1}$ has been found. Such a value is located in the non-acceptability zone for what concern the societal risk $(<100$ inhabitants) and at the boundary of the acceptability region if an individual risk is considered.

\section{Conclusions}

Analysing the two fault trees generated for the undesired event "explosion in the de-dusting section of a cement plant", it has been showed that the probabilities of occurrence of the same top event (considered a mission time equal to 1 year) are, respectively, $5.6 \cdot 10^{-8} \mathrm{y}^{-1}$ and $3.8 \cdot 10^{-6} \mathrm{y}^{-1}$, before and after the performing of a retrofit operation consisting in the addition of a fabric filter downstream an electrostatic precipitator (in order to improve the de-pulverisation performances of the unit to meet with new more stringent regulations on threshold dust emission values).

While in the first configuration the risk can be considered acceptable (and the system does not require to be integrated with redundant protection devices in order to improve the reliability of the de-dusting section), in the retrofit configuration the risk follows within the non acceptability zone. Therefore, the system requires to be integrated with redundant protection devices in order to improve the reliability of the retrofit de-dusting section.

This simple study confirms that each process or piece of equipment change must be fully evaluated from the safety and reliability viewpoint before being introduced into an already existing plant because it can imply the increase of the risk function.

\section{References}

[1] Explosions in Cement Plants. www.fike.com/

[2] http://www.greenreport.it/

[3] Rada, E.C., Squazardo, S., Ionescu, G., Badea, A. Economic viability of SRF co-combustion in cement factory, UPB Scientific Bulletin, series D, 76(3), pp. 199-206, 2014.

[4] Ragazzi, M., Rada, E.C. RDF/SRF evolution and MSW bio-drying, WIT Transactions on Ecology and the Environment, 163, pp. 199-208, 2012.

[5] Cioranu, S.I., Grigoriu, M., Ragazzi, M., Rada, E.C., Ionescu, G. Assessment of alternatives for RMSW treatment for Valcea County, Romania, Revista de Chimie, 65(3), pp. 257-261, 2014.

[6] Sarc, R., Lorber, K.E., Opmberger, P., Rogetzer, M., Sipple, E.M. Design, quality, and quality assurance of solid recovered fuels for the substitution of fossil feedstock in the cement industry, Waste Management and Research, 32(7), pp. 565-585, 2014.

[7] Taiwo, A.M., Harrison, R.M., Shi, Z. A review of receptor modelling of industrially emitted particulate matter, Atmospheric Environment, 97, pp. 109-120, 2014 
[8] Torretta, V., Rada, E.C., Panaitescu, V., Apostol, T. Some consideration on particulate generated by traffic, UPB Scientific Bulletin, series D, 74(4), pp. 214-248, 2012.

[9] Seltenrich, N. Take care in the kitchen: Avoiding cooking-related pollutants, Environmental Health Perspectives, 122(6), pp. A155-A159, 2014.

[10] Sari, D., Bayram, A. Turkey and assessment of the impact on local/regional air-quality, Science of the Total Environment, 488-489(1), pp. 429-436, 2014.

[11] Capodaglio, A., Callegari, A., Torretta, V. Comparative application of different risk assessment models and implications on resulting remediation options, Environmental Technology 35(6), pp. 759-765, 2014.

[12] Hansler, R.J., Gooijer, L., Wolting, B.G. Acute inhalation toxicity in quantitative risk assessment-methods and procedures, Chemical Engineering Transactions, 31, pp. 751-756, 2013.

[13] Vaseley, W.E., Goldberg, F.F., Roberts, N.H., Haasl, D.F., Fault Tree Handbook, U.S. Nuclear Regulatory Commission Washington D.C., 1981.

[14] Dossier Ambiente, http://www.amblav.it/dossier.aspx 